\title{
Unequal Error Protection SCMA Codebooks
}

\author{
Zeina Mheich, Lei Wen, Pei Xiao and Amine Maaref
}

\begin{abstract}
This paper investigates the design of unequal error protection (UEP) codebooks for sparse code multiple access (SCMA) systems. We propose a joint LDPC code and SCMA codebook design approach by incorporating cloud-partitioning of codewords in the design of SCMA codebooks with different protection levels. The protection levels of the SCMA codebooks could be optimized based on the existing error correction code. Simulation results show that significant gains could be obtained using code-aware UEP SCMA codebooks compared to codebooks designed independently of the channel code.
\end{abstract}

Index Terms-NOMA, SCMA, UEP, codebook design, channel coding.

\section{INTRODUCTION}

Non-orthogonal multiple access (NOMA) techniques support massive connectivity and improve the spectral efficiency of wireless radio access by enabling overloading (i.e., the number of multiplexed users can be larger than the number of resources). Low-density signature (LDS) is a code-domain NOMA where the constellation symbol is expanded to a sequence of complex symbols by using a specific signature [1]. The low-density characteristic of LDS signatures allows to use message passing algorithm for multi-user detection with lower complexity than maximum-likelihood decoding. SCMA is a generalization of LDS, where the procedure of bit to modulation mapping and spreading are combined together such that coded bits are directly mapped to multi-dimensional sparse codewords selected from user-specific SCMA codebooks [2]. By using multi-dimensional codebooks, SCMA can benefit from shaping gains of multi-dimensional constellations. There are many SCMA codebook design methods in literature. Nikopour et al. are the first to propose a multi-stage suboptimal method for the design of multiuser SCMA codebooks [3]. Following this direction, other sub-optimal design methods are proposed e.g. in [4], [5], [6], [7], [8] to improve the performance or to lower the complexity of multiuser detection or codebook design. Most these works assess the performance of codebooks in uncoded systems or do not take into account the error-correction code in their codebook design. In a real communication system, an error-correction code is used and

Copyright (c) 2019 IEEE. Personal use of this material is permitted. However, permission to use this material for any other purposes must be obtained from the IEEE by sending a request to pubs-permissions@ieee.org.

This work was sponsored by Huawei Technologies Co., Ltd, Ottawa, Canada. It was also supported in part by the UK Engineering and Physical Sciences Research Council (EPSRC) under grant number EP/P03456X/1.

Zeina Mheich and Pei Xiao are with the 5G Innovation Centre, Institute for Communication Systems, University of surrey, U.K. (e-mail: \{z.mheich, p.xiao\}@ surrey.ac.uk)

Lei Wen is with the Department of Communication Engineering, College of Electronic Science and Engineering, National University of Defense Technology, Changsha, China. (e-mail: newton1108@126.com)

Amine Maaref is with the Canada Research Center, Huawei Technologies Company Ltd., Ottawa, ON, Canada. (e-mail: Amine.Maaref@huawei.com) may introduce unequal protection between coded bits (i.e. some bits are more protected than others).

This paper proposes to design UEP codebooks for SCMA systems. The proposed design method follows a joint LDPC code and SCMA codebook design approach and is based on cloud partitioning of the SCMA codewords. We also introduce a particular design method of UEP SCMA codebooks based on constellation rotation and interleaving. This algorithm is based on applying cloud-partitioning to the codebooks proposed in [6]. Finally, we propose to optimize the protection levels based on the $5 \mathrm{G}$ compliant channel code.

\section{SCMA SYSTEM MODEL}

We consider a SCMA system where $V$ users are sharing $K$ orthogonal resources, e.g. OFDMA sub-carriers. SCMA enables overloading such that the number of users can be more than the number of resources $V>K$. The overloading factor is defined as $\lambda=V / K$. Each user has a multi-dimensional SCMA codebook consisting of $M$ codewords where each codeword occupies $K$ resources. An SCMA encoder for user $u$ is defined as a mapping ${ }^{1}$

$$
f_{u}: \mathbb{B}^{\log _{2}(M)} \rightarrow \mathcal{C}_{u},
$$

where $\mathcal{C}_{u} \subset \mathbb{C}^{K}$ is the codebook of user $u$ with cardinality $M$. A vector $\mathbf{b}$ of $\log _{2}(M)$ coded bits is mapped to a multidimensional codeword $\mathbf{c}=f_{u}(\mathbf{b})$ selected from $\mathcal{C}_{u}$. Each complex codeword c is a sparse vector with $N<K$ nonzero entries. The sparsity of the codewords enables to limit the number of users colliding over the same resource which in turn reduces the complexity of user detection. Let $\mathbf{x}$ denote the vector of non-zero complex symbols obtained by removing the zeros from the codeword $\mathbf{c}$. The vector $\mathbf{x}$ can be interpreted as an $N$-dimensional complex constellation point belonging to a multi-dimensional constellation $\mathcal{X}_{u} \subset \mathbb{C}^{N}$. The mapping from $\mathbb{B}^{\log _{2}(M)}$ to $\mathcal{X}_{u}$ is defined as,

$$
g_{u}: \mathbb{B}^{\log _{2}(M)} \rightarrow \mathcal{X}_{u},
$$

such that $\mathbf{x}=g_{u}(\mathbf{b})$. Then the SCMA encoder in (1) can be redefined as $f_{u}: \equiv \mathbf{V}_{u} \cdot g_{u}$ where $\mathbf{V}_{u} \in \mathbb{B}^{K \times N}$ is the binary mapping matrix which maps the $N$-dimensional constellation point to a $K$-dimensional SCMA codeword. The mapping matrix $\mathbf{V}_{u}$ contains $K-N$ all-zero rows. By eliminating the $K-N$ all-zero rows from $\mathbf{V}_{u}$, an identity matrix $\mathbf{I}_{N}$ of size $N \times N$ is obtained. A row $r \in\{1, \cdots, K\}$ containing a value 1 in $\mathbf{V}_{u}$ means that user $u$ is using the $r^{\text {th }}$ resource. Hence, the set of resources occupied by user $u$ depends on $\mathbf{V}_{u}$.

An SCMA code can be represented by
$\mathcal{S}\left(\left[\mathbf{V}_{u}\right]_{u=1}^{V},\left[g_{u}\right]_{u=1}^{V} ; V, M, N, K\right)$. The whole structure

\footnotetext{
${ }^{1}$ Notations: throughout this paper, $\mathbb{B}, \mathbb{C}, \mathbb{Z}$ denote respectively the set of binary, complex and integer numbers.
} 


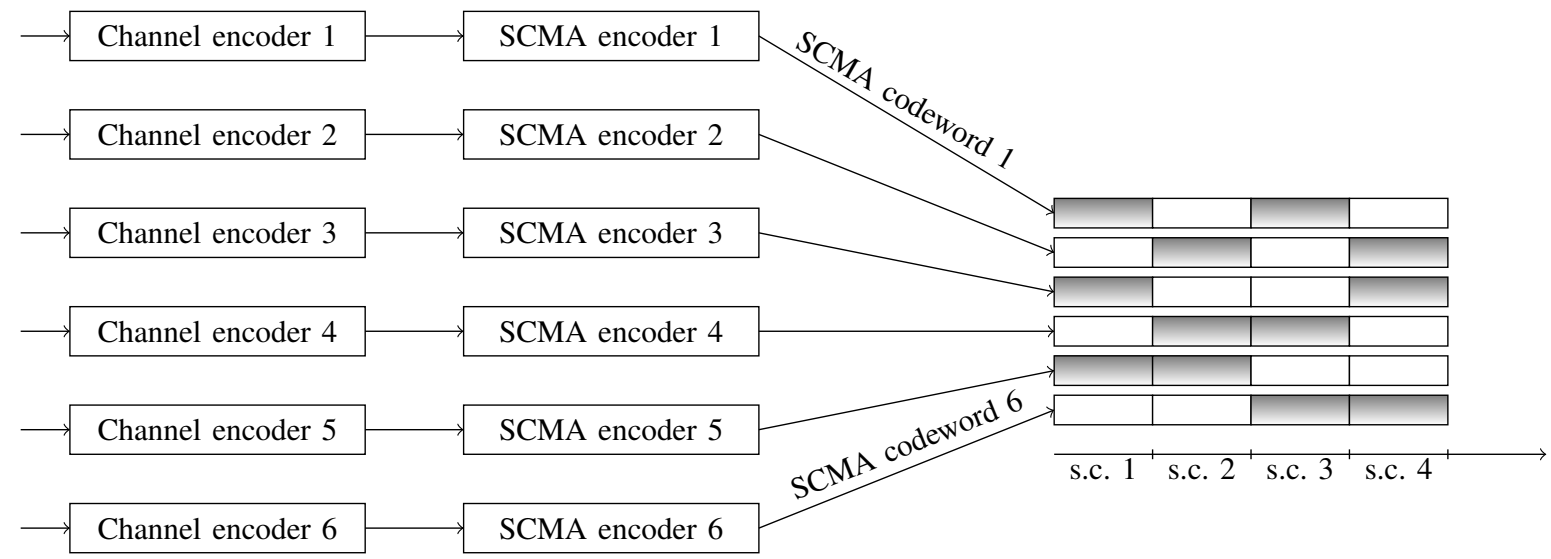

Figure 1. Block diagram of the SCMA system represented by the factor graph matrix in (3).

of SCMA code $\mathcal{S}$ can be represented by a factor graph matrix $\mathbf{F}=\left(\mathbf{f}_{1}, \cdots, \mathbf{f}_{V}\right) \subset \mathbb{B}^{K \times V}$ where $\mathbf{f}_{u}=\operatorname{diag}\left(\mathbf{V}_{u} \mathbf{V}_{u}^{T}\right)$. The resource $k$ is used by user $u$ if and only if $F_{k u}=1$, where $F_{k u}$ is the element in the $k$ th row and $u$ th column of $\mathbf{F}$.

Example: Consider a SCMA system of $J=6$ users and $K=4$ resources. The factor graph matrix is given in equation (3). Each user occupies $N=2$ resources (number of ones in each column of $\mathbf{F}$ ) and each resource is used by 3 users (number of ones in each row of $\mathbf{F}$ ). The corresponding SCMA system is shown in Figure 1, where each SCMA codeword occupies 4 subcarriers (s.c.). For each user, the SCMA encoder maps each $\log _{2}(M)$ coded bits obtained from the channel encoder to a SCMA codeword chosen from its codebook of size $M$. The non-zero values of each codeword are filled in gray and can be obtained directly from $\mathbf{F}$.

$$
\mathbf{F}=\left[\begin{array}{llllll}
1 & 0 & 1 & 0 & 1 & 0 \\
0 & 1 & 0 & 1 & 1 & 0 \\
1 & 0 & 0 & 1 & 0 & 1 \\
0 & 1 & 1 & 0 & 0 & 1
\end{array}\right]
$$

\section{UEP SCMA CODEBOOK}

This section introduces the cloud partitioning method used to construct UEP SCMA codebooks. A general implementation of cloud partitioning which could be incorporated in existing codebook design is presented. It is followed by a particular implementation of UEP SCMA codebooks based on constellation rotation and interleaving.

\section{A. Cloud partitioning}

We propose to design a UEP SCMA codebook $\mathcal{C}$ of size $M=2^{m}$ with $L \leq m$ protection levels. We assign to each codeword $\mathbf{c}_{i}(i=1, \cdots, M)$ a binary label vector $\left(b_{0}^{i}, b_{1}^{i}, \cdots, b_{m-1}^{i}\right), b_{j}^{i} \in\{0,1\}$. The method used to provide UEP for the bits $b_{j}^{i}$ is based on cloud partitioning of codewords. In the following, we list the steps used toward a general implementation of cloud partitioning.

- We assign to each bit $b_{j}(j=0,1, \cdots, m-1)$ of the binary label vector $\left(b_{0}, b_{1}, \cdots, b_{m-1}\right)$ of the codebook, one protection level $\ell \in \mathcal{L}=\{1, \cdots, L\}$. We denote by
$\mathcal{B}_{\ell}$ the set of the bits having the same protection level $\ell$. We assume that $\mathcal{B}_{\ell} \neq \emptyset$ and $\mathcal{B}_{i} \cap \mathcal{B}_{j}=\{\emptyset\}, \forall i, j, l \in \mathcal{L}$.

- Let $M_{\ell}=2^{\left|\mathcal{B}_{\ell}\right|}$ where |.| is the cardinality. For each level $\ell, M_{\ell}$ "clouds" $\mathcal{C} \ell_{i}\left(i=1 \cdots M_{\ell}\right)$ are generated such that the minimum distance between two clouds in the same level is equal to $d_{\text {min }}^{\ell}$. Each cloud $\mathcal{C} \ell_{i}$ has a binary label vector $s_{i}^{\ell}$ which represents a special realization of the set $\mathcal{B}_{\ell}$. For example, consider $\mathcal{B}_{1}=\left\{b_{i}, b_{j}\right\}$, we generate 4 clouds for level 1 , the four clouds $\mathcal{C} \ell_{i}, i=1, \cdots, 4$ are labeled $s_{1}^{1}=\{00\}, s_{2}^{1}=\{01\}, s_{3}^{1}=\{10\}, s_{4}^{1}=\{11\}$ respectively. Let $\mathcal{B}_{\ell}=\left\{b_{i}, b_{j}\right\}$; each codeword in $\mathcal{C}$ with $\left\{b_{i}, b_{j}\right\}=s_{k}^{\ell}$ belongs to the $k$ th cloud $\mathcal{C} \ell_{k}$ of level $\ell$.

- Without loss of generality, we assume that the protection level decreases from 1 to $L$; i.e., the bits in $\mathcal{B}_{\ell}$ are more protected than those in $\mathcal{B}_{\ell+1}$. Hence the clouds are generated such that $d_{\text {min }}^{\ell}>d_{\text {min }}^{\ell+1}, \forall \ell \in \mathcal{L}$ which leads to unequal error protection between clouds that do not belong to the same level. Moreover, for each level $\ell$, the minimum distance between codewords in the same cloud should be less than $d_{m i n}^{\ell}$. Under these constraints, $d_{\text {min }}^{\ell}$ is optimized to improve the system performance. Specifically, we propose to optimize $d_{\min }^{\ell}$ based on the existing error correction code.

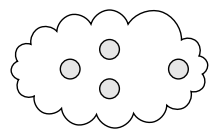

Cloud $\mathcal{C} \ell_{1}, s_{1}=\{00\}$

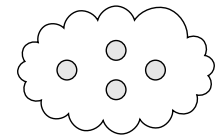

Cloud $\mathcal{C} \ell_{2}, s_{2}=\{01\}$

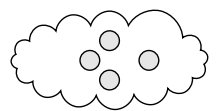

Cloud $\mathcal{C} \ell_{4}, s_{4}=\{10\}$

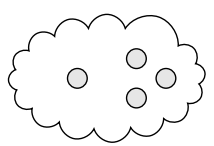

Cloud $\mathcal{C} \ell_{3}, s_{3}=\{11\}$
Figure 2. Illustration of UEP SCMA codebook with two protection levels and 16 codewords.

Figure 2 illustrates the cloud-partitioning principle for a codebook of $M=16$ codewords with two protection lev- 
els. The shaded circles represent codewords. The codewords belonging to a cloud $\mathcal{C} \ell_{i}$ have a binary label vector starting with $s_{i}$, e.g., codewords in cloud 1 have a labeling vector $\left(0,0, b_{2}, b_{3}\right)$. The codewords belonging to different clouds are more distant from each other than codewords inside the same cloud. This gives more protection for the bits $b_{0}, b_{1}$ assigned to the clouds than bits $b_{2}, b_{3}$ assigned to the codewords inside the same cloud.

A practical implementation of cloud partitioning to build a codebook with $L$ protection levels is to superpose $L$ codebooks with different average power. For example, for 1-dimensional codebooks, this is equivalent to superpose two constellations with different average power. The superposition of two 4quadrature amplitude modulation (QAM) constellations, of average powers $P_{1}$ and $P_{2}$ respectively, produces a 16-QAM with two protection levels where the levels can be adjusted by the ratio $\frac{P_{1}}{P_{2}}$. A well-known example of a 1-dimensional UEP constellation is the hierarchical modulation [9] included in various standards.

The cloud-partitioning method described above could be incorporated in existing SCMA codebook design to create UEP SCMA codebooks (e.g. star-QAM codebooks [7], golden angle modulation (GAM)-based codebooks [8], $\cdots$ ). In the sequel, we show how to include cloud-partitioning in the codebook design proposed in [6]. Note that the construction of UEP SCMA codebooks based on GAM, star-QAM or others could be conducted in the same manner.

\section{B. Particular design method based on constellation rotation and interleaving}

This section provides a particular implementation of UEP SCMA codebooks with cloud partitioning. It is based on the superposition of codebooks obtained using the scheme proposed in [6]. The advantage of the codebook design in [6] is that it allows to construct codebooks for SCMA systems with arbitrary number of users, resources and codewords in a simple manner. Note that this implementation is more efficient in the downlink. In [6], the codebook design method consists of constructing first a mother constellation of dimension $N$ with $M$ points. Then, the codebook for each user is obtained by applying a constellation operator to the mother constellation. However, we assume here that the users do not necessarily have the same mother constellation. In the following, we show how to modify the codebook designed in [6] to create UEP codebooks.

For each user $u$ the following steps are employed:

- For each level $\ell$, define the row vector $\mathbf{t}^{\ell}=$ $\left[t_{1}^{\ell}, t_{2}^{\ell}, \cdots, t_{M_{\ell}}^{\ell}\right]$, where $t_{m}^{\ell}=\left(2 m-1-M_{\ell}\right) \cdot(1+i)$, $m=1, \cdots, M_{\ell}$ and $i=\sqrt{-1}$. Note that $\mathbf{t}^{\ell}$ represents a 1-dimensional lattice of equally spaced complex symbols.

- For each level $\ell$, define the amplitude row vector $\mathbf{a}^{\ell, u}=$ $\left[a_{1}^{\ell, u}, \cdots, a_{N}^{\ell, u}\right]$. Since we assume that level $\ell$ is more protected than level $j$ if $\ell<j$, we have $a_{n}^{\ell, u}>a_{n}^{j, u}$ for each dimension $n \in\{1, \cdots, N\}$. $\mathbf{a}^{\ell, u}$ is a new parameter added to the design method of [6].

- For each $n \in\{1, \cdots, N\}$, construct the 1-dimensional lattice $\mathbf{r}^{n, u}$ of $M$ complex symbols by superposing $L$ constellations $a_{n}^{1, u} \mathbf{t}^{1} \boxplus \cdots \boxplus a_{n}^{L, u} \mathbf{t}^{L}$ where $\boxplus$ stands for constellation superposition.

- Build a matrix $\Omega^{u}$ of size $N \times M$. The $n$th row of $\Omega^{u}$ is given by $\mathbf{r}^{n, u} e^{i \theta_{n}}$, where $\theta_{n}=(n-1) \frac{\pi}{M N}$.

- Construct the mother constellation $\mathbf{M}_{c}^{u}$ for user $u$, by interleaving the elements of some rows of $\Omega^{u}$. For example, in [6] the authors propose an interleaver for the rows of even indices. The same interleaver could be used to design of UEP codebooks, however, care should be taken not to interleave two clouds at level $\ell+1$ belonging to different clouds of level $\ell$. Hence, at level 1 , we could interleave clouds between them and for $\ell>1$ we could only interleave clouds belonging to the same mother cloud at level $\ell+1$.

- Apply a rotation for each dimension of $\mathbf{M}_{c}^{u}$ depending on user index, in order to increase the diversity between codebooks. A simple method was given in [6] in order to construct the final codebook for each user from its mother codebook. Let $N_{r}$ be the number of users colliding over the same resource and $\phi_{r}=(r-1) \frac{2 \pi}{M N_{r}}+\frac{2 \pi}{M} e_{r}$ for $r=1, \cdots, N_{r}$, where $e_{r}$ is an arbitrary member of $\mathbb{Z}$. We build a Latin matrix $\mathbf{L}$, by replacing the non-zero elements of $\mathbf{F}$ by $e^{i \phi_{r}}$, where the $\phi_{r}$ are chosen such that $\mathbf{L}$ is Latin. For each user $u$, we define $\boldsymbol{\Delta}_{u}=\operatorname{diag}\left(\mathbf{L}_{u}\right)$, where $\mathbf{L}_{u}$ is the $u$ th column of $\mathbf{L}$ without zero elements, and $\mathbf{V}_{u}$ the mapping matrix of size $K \times N$. Then the codebook of user $u$ is given by $\mathcal{C}_{u}=\mathbf{V}_{u} \cdot \boldsymbol{\Delta}_{u} \cdot \mathbf{M}_{c}^{u}$.

Usually, UEP constellations are used to provide UEP to different classes of data having different priorities [9]. In addition, UEP codebooks can be utilized to optimize the power allocated to the coded bits based on the existing channel code as shown in the next section.

\section{Optimization of UEP SCMA codebooks for LDPC codes}

This section shows how to optimize the UEP SCMA codebooks when a low-density parity check (LDPC) code is used as a channel code. In irregular LDPC codes, the reliability of variable nodes after decoding depends on their degrees. During message passing decoding, variable nodes of higher degrees converge faster than those of lower degrees. Moreover, since variable nodes of higher degrees are well connected to the LDPC graph, their messages dominate in the graph. In [10], a bit-reliability mapping is proposed for LDPC-coded modulation systems. The principle of bit-reliability mapping is to map the less reliable LDPC code bits to the lower level modulation bits and the more reliable bits to the higher level modulation bits.

We propose to use bit-reliability mapping for LDPC-coded SCMA systems. Thus, the variable nodes of higher degrees are assigned to the higher level bits of the UEP SCMA codebook and the variable nodes of lower degrees to the lower level bits. The bit-reliability mapping is implemented with the aid of an interleaver between the LDPC encoder and the SCMA mapper. Finally, the UEP codebook should be optimized based on the variable node degree distribution of the LDPC code in order to improve the error rate performance. By using bit-reliability mapping together with UEP SCMA codebooks, more power could be allocated to the variable nodes of higher degrees. 


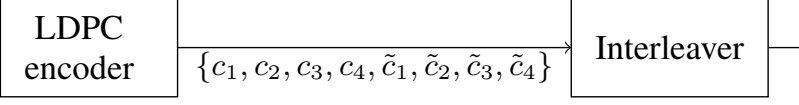

\begin{tabular}{l|l} 
SCMA \\
mapper
\end{tabular}$\left\{c_{1}, c_{2}, \tilde{c}_{1}, \tilde{c}_{2}, c_{3}, c_{4}, \tilde{c}_{3}, \tilde{c}_{4}\right\}$

Figure 3. Block diagram of LDPC-coded SCMA system.

Example: In Figure 3, assume that the UEP SCMA codebook of Figure 2 is used. The LDPC encoder produces a codeword of 8 bits such that bits $c_{i}, i=1, \cdots, 4$ are assigned to variable nodes of higher degrees than bits $\tilde{c}_{i}$. The output of the interleaver is such that bits $c_{i}$ are mapped to the high protection level of the SCMA codebook in Figure 2 and bits $\tilde{c}_{i}$ are mapped to the low protection level of the SCMA codebook. In this way, the first two bits of the label vector carry variable nodes of high degrees. Thus, more power is allocated to higher degree variable nodes. The power allocation is optimized based on the variable node degree distribution of the LDPC code.

\section{RESUlTS AND DISCUSSIONS}

We design two-dimensional UEP SCMA codebooks of 16 points with 2 protection levels for 6 users sharing 4 resources. Each codeword has a binary label vector of 4 bits $\left(b_{0}, b_{1}, b_{2}, b_{3}\right)$. The factor matrix is given in (3).

We use binary LDPC codes as channel codes, chosen from 3GPP standard for 5G [11]. The same interleaver adopted in [11] is employed between channel encoder and SCMA codebook mapper ${ }^{2}$. At the receiver, the message passing algorithm for multi-user detection performs 6 iterations. The LDPC decoder performs 20 iterations using sum-product decoding. There are no outer iterations between the multi-user detector and channel decoder.

Figure 4 compares the average error rate performance of the UEP SCMA codebooks obtained using the design principle of Sec. III-B (where $\mathbf{a}^{\ell, u}$ are optimized based on the LDPC code) and the benchmark SCMA codebooks of [6] ( $\mathbf{a}^{\ell, u}$ are fixed) for the downlink Rayleigh fading channel. The bit error rate (BER) and the frame error rate (FER) are calculated on systematic bits only (uncoded bits). It can be observed that the gain obtained by optimizing the vectors $\mathbf{a}^{\ell, u}$ increases when the code rate decreases for the LDPC codes in [11]. Hence the codebook should be adapted to the code rate. For a code rate $1 / 3$ and frame length 132 bits, a gain up to $2.5 \mathrm{~dB}$ is achieved. For a code rate $2 / 5$ and frame length 550 bits, a gain up to $1.5 \mathrm{~dB}$ is achieved. For a code rate $1 / 2$ and frame length 440 bits, a gain up to $0.5 \mathrm{~dB}$ is observed for target FER greater than $4 \times 10^{-3}$. The vectors $\mathbf{a}^{\ell, u}$ given by the benchmark codes become optimal for target FER lower than $4 \times 10^{-3}$. It should be noted that the amplitude row vectors $\mathbf{a}^{\ell, u}$ are not equal for all users in order to further increase the power diversity between users colliding over the same resource. However, they

\footnotetext{
${ }^{2}$ The interleaver adopted in [11] maps the bits $b_{0}, b_{1}$ to variable nodes of degrees higher than or equal to the variable nodes mapped to bits $b_{2}, b_{3}$.
}

could be chosen to be equal in order to lower the optimization complexity.

The peak to average power ratios (PAPR) of the optimized UEP SCMA codebooks are given in Table I. It can be observed that optimizing the UEP SCMA codebook also results in lower PAPR.

\begin{tabular}{|c|c|c|c||c|}
\hline Rate & $1 / 3$ & $2 / 5$ & $1 / 2$ & Ref. [6] \\
\hline \hline PAPR [dB] & 0.2798 & 0.5921 & 1.0397 & 1.2366 \\
\hline
\end{tabular}

Table I

PAPR OF THE OPTIMIZED UEP SCMA CODEBOOKS FOR DIFFERENT CODE RATES AND OF THE BENCHMARK CODEBOOKS IN [6].

In this paper, LDPC codes are chosen from 3GPP standard for 5G. In general, the LDPC code, the mapper and the protection levels of the UEP SCMA codebook could be optimized jointly using EXIT charts. The sparse graphs of LDPC code and SCMA could also be combined into one joint sparse graph which improves the overall performance of the system [12]. However, in practice, it is desirable to design an LDPC code which can support multiple rates and multiple codebook/constellation sizes within the context of bit-interleaved coded systems [13] instead of optimizing the LDPC code for each codebook/constellation size.

\section{CONCLUSION AND FUtURE WORK}

We studied the design of UEP SCMA codebooks based on cloud partitioning and proposed to optimize the protection levels of the codebooks based on the channel code. Simulation results for LDPC-coded SCMA systems showed that optimizing UEP SCMA codebooks based on this joint design approach could enhance the error rate performance and lower the PAPR.

The extension to other NOMA techniques is straightforward. The cloud-partitioning method in Sec. III-A can be used to design UEP constellations for power-domain NOMA and LDS with different protection levels. A practical method to design a constellation with $\tilde{L}$ protection levels is to superpose $\tilde{L}$ constellations with different average powers. The average powers can be optimized based on the existing channel code.

\section{REFERENCES}

[1] R. Hoshyar, F. P. Wathan, and R. Tafazolli, "Novel low-density signature for synchronous CDMA systems over AWGN channel," IEEE Transactions on Signal Processing, vol. 56, no. 4, pp. 1616-1626, 2008.

[2] H. Nikopour and H. Baligh, "Sparse code multiple access," in IEEE PIMRC, 2013, pp. 332-336.

[3] M. Taherzadeh, H. Nikopour, A. Bayesteh, and H. Baligh, "SCMA codebook design," in IEEE VTC Fall, 2014, pp. 1-5.

[4] J. Bao, Z. Ma, M. A. Mahamadu, Z. Zhu, and D. Chen, "Spherical codes for SCMA codebook," in IEEE VTC Spring, 2016, pp. 1-5.

[5] Y. Zhou, Q. Yu, W. Meng, and C. Li, "SCMA codebook design based on constellation rotation," in IEEE ICC, 2017, pp. 1-6.

[6] D. Cai, P. Fan, X. Lei, Y. Liu, and D. Chen, "Multi-dimensional SCMA codebook design based on constellation rotation and interleaving," in IEEE VTC Spring, 2016, pp. 1-5.

[7] L. Yu, X. Lei, P. Fan, and D. Chen, "An optimized design of SCMA codebook based on star-QAM signaling constellations," in WCSP, 2015.

[8] Z. Mheich, L. Wen, P. Xiao, and A. Maaref, "Design of SCMA Codebooks Based on Golden Angle Modulation," IEEE Transactions on vehicular technology, accepted for publication. 


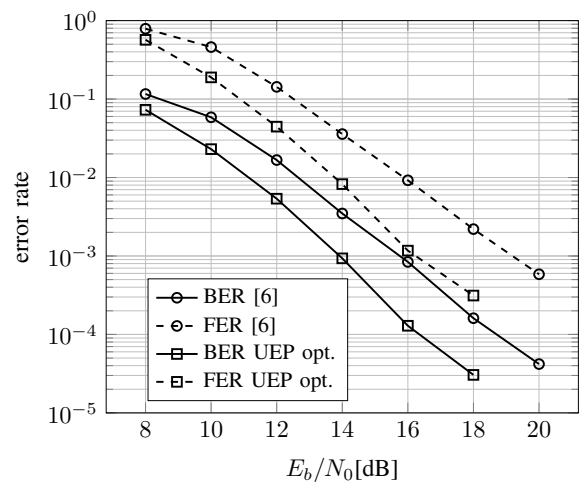

(a)

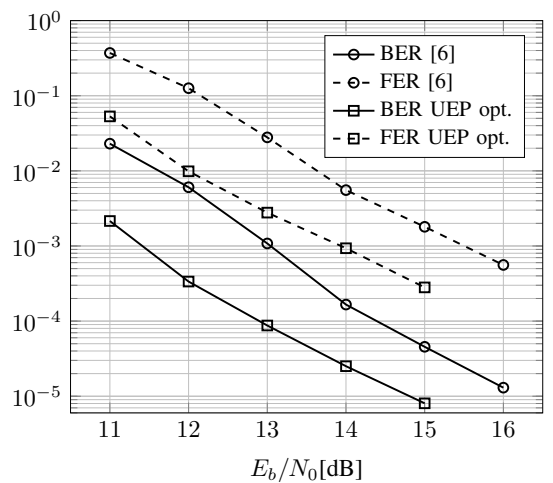

(b)

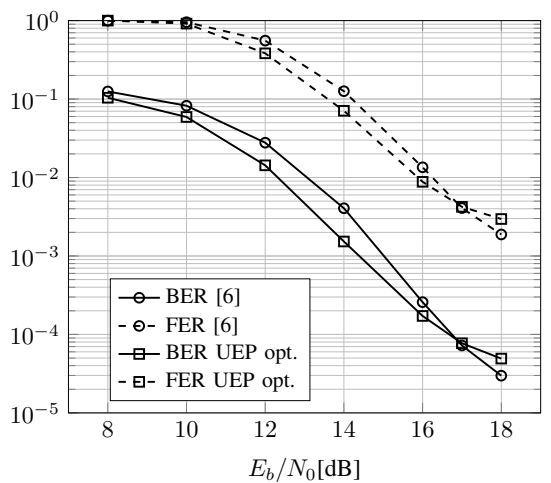

Figure 4. Average error rate in the downlink Rayleigh fading SCMA channel. (a) $\mathrm{R}=1 / 3$, frame length = 132 bits; (b) $\mathrm{R}=2 / 5$, frame length $=550$ bits; (c) $\mathrm{R}=1 / 2$, frame length $=440$ bits.

[9] H. Jiang and P. A. Wilford, "A hierarchical modulation for upgrading digital broadcast systems," IEEE Transactions on Broadcasting, vol. 51, no. 2, pp. 223-229, June 2005.

[10] Y. Li and W. E. Ryan, "Bit-reliability mapping in LDPC-coded modulation systems," IEEE Communications Letters, vol. 9, no. 1, pp. 1-3, Jan 2005.

[11] 3GPP, "TS38.212 Multiplexing and channel coding," 2018. [Online]. Available: http://www.3gpp.org/ftp//Specs/archive/38_series/38.212/

[12] K. Lai, L. Wen, J. Lei, P. Xiao, A. Maaref, and M. A. Imran, "Sub-Graph Based Joint Sparse Graph for Sparse Code Multiple Access Systems," IEEE Access, vol. 6, pp. 25 066-25 080, 2018.

[13] T. V. Nguyen, A. Nosratinia, and D. Divsalar, "Threshold of ProtographBased LDPC Coded BICM for Rayleigh Fading," in IEEE GLOBECOM 2011, Dec 2011, pp. 1-5. 